\title{
Tailoring Nanocomposite Membranes of Cellulose of Acetate/Silica Nanoparticles for Desalination
}

\section{Amira S. Mohammed Ali ( $\square$ amira.mali@alexu.edu.eg )}

Alexandria University

Moataz M. Soliman

Alexandria University

Sherif H. Kandil

Alexandria University

Shaker Ebrahim

Alexandria University

Marwa Khalil

City of Scientific Research and Technological Applications (SRTA-City)

\section{Research Article}

Keywords: Silica nanoparticles, Amino-modified silica, APTES, Cellulose acetate, Nanocomposite membrane, Water desalination

Posted Date: August 23rd, 2021

DOI: https://doi.org/10.21203/rs.3.rs-820853/v1

License: (c) (i) This work is licensed under a Creative Commons Attribution 4.0 International License.

Read Full License 


\title{
Tailoring Nanocomposite Membranes of Cellulose of Acetate/Silica Nanoparticles for Desalination
}

\author{
Amira S. Mohammed Alia ${ }^{a}$, Moataz M. Soliman ${ }^{a}$, Sherif H. Kandila, Shaker Ebrahim \\ Marwa Khalil ${ }^{b}$ \\ ${ }^{a}$ Materials Science Department, Institute of Graduate Studies and Research (IGSR), Alexandria \\ University, 163 Horrya Avenue, El-Shatby, P.O. Box 832, Postal Code: 21526, Alexandria, Egypt. \\ ${ }^{\mathrm{b}}$ Nanotechnology and Composite Materials Department, Institute of New Materials and Advanced \\ Technology, City of Scientific Research and Technological Applications (SRTA-City), P.O. Box \\ 21934, New Borg El Arab City, Alexandria, Egypt. \\ *Corresponding author: Materials Science Department, Institute of Graduate Studies and Research \\ (IGSR), Alexandria University, 163 Horrya Avenue, El-Shatby, P.O. Box 832, Postal Code: \\ 21526, Alexandria, Egypt. Email: amira.mali@alexu.edu.eg
}

\begin{abstract}
Herein, fabrication of cellulose acetate (CA) silica-based nanocomposite membranes via the drywet phase inversion procedure for the water desalination was investigated. The modified and unmodified silica nanoparticles (SNPs and MSNPs) were prepared by the sol-gel technique. The addition effect of the SNPs and MSNPs was investigated on the CA membranes properties and their performance for water separation. The CA nanocomposite membranes were characterized to study their structure, hydrophilicity, and morphology. The fabricated nanocomposite membranes showed hydrophilic surface properties. The performance of reverse osmosis (RO) membranes was measured using a crossflow RO unit. At $10 \mathrm{bar}$, The membrane with $10 \mathrm{mg}$ of SNPs showed enhanced permeate water flux compared to the pristine CA membrane by $1.6 \mathrm{~L} / \mathrm{m}^{2} . \mathrm{hr}$. Increasing the SNPs in the nanocomposite membrane showed enhancement in the permeate water flux all over the operating pressure. The effect of MSNPs on the nanocomposites' performance was lower than their counterpart in the case of adding SNPs. The membrane with $30 \mathrm{mg}$ of MSNPs showed the highest permeate water flux among other nanocomposite membranes with a value of 35.7 $\mathrm{L} / \mathrm{m}^{2}$.hr at 24 bar.
\end{abstract}

Keywords: Silica nanoparticles, Amino-modified silica, APTES, Cellulose acetate, Nanocomposite membrane, Water desalination. 


\section{Introduction}

Desalination is now utilized to resolve freshwater shortages in many parts of the world ${ }^{1}$. It is a feasible opportunity that can reduce the gap between the accelerating demands and the available capacities. That has been used for quite in many rural, deserted areas and coastal regions to provide clean drinking water ${ }^{2}$. The traditional solutions are being demonstrated by the most recent commercial technologies such as reverse osmosis (RO), multi-stage flash desalination, and multieffect distillation ${ }^{1}$. RO is widely accepted and applied as an environmentally friendly and effective method for desalting numerous types water; for example, groundwater, rivers, lakes, brackish water, and seawater ${ }^{3}$.

Polymeric membranes either as a support or an active material are used widely in RO applications

${ }^{4}$. Different methods to produce efficient and low-cost biopolymers for water desalination have recently been investigated. $\mathrm{CA}$ is one of the most promising biopolymers which increased the hydrophilic character, excellent selectivity, toughness, ease to fabricate, and have resistance to degradation by chlorine ${ }^{5,6}$. However, CA membranes suffer from low flux, prone to compaction (loss of permeability at high pressure), and hydrolysis in alkaline and acidic medium ${ }^{7}$.

Nanomaterials and nanoparticles consider are suitable additives to enhance membrane performance such as selectivity and permeability, fouling resistance, hydrophilicity, and inhibit macrovoid formation ${ }^{8-10}$. Several studies have investigated the effect of nanoparticles in the polymeric membrane such as zeolite ${ }^{11,12}$, carbon nanotubes ${ }^{13}$, graphene oxide ${ }^{14}$, alumina $\left(\mathrm{Al}_{2} \mathrm{O}_{3}\right)$ ${ }^{15}$, clay ${ }^{16}$, and titania ${ }^{17}$. Moreover, the addition of the inorganic nanoparticles to the membrane can serve to induce an increase in the mean distance (free volume) between polymer chains and restrict the molecular motions of the polymer chains which can lead to simultaneous improvement in both selectivity and permeability ${ }^{18}$. The major drawback of the addition of the nanoparticles is their strong tendency to agglomerate followed by insufficient dispersal in the membrane ${ }^{19}$. Recent solutions for the agglomeration of nanoparticles in polymeric membranes include surface modification and optimization of the fabrication processes ${ }^{20}$.

The addition of SNPs to CA membranes has been studied before to investigate their effect on CA membranes using polyethylene glycol-600 as a pore-forming agent. The membrane preparation was done using a 2-stage phase inversion procedure by Ahmad, A., et al ${ }^{21}$. The flux enhanced from 0.35 to $2.46 \mathrm{~L} / \mathrm{h} . \mathrm{m}^{2}$ with an $11.41 \%$ relative increase in the salt rejection. Also, they have prepared membranes from CA, polyethylene glycol 300 conjugated with SNPs by thermallyinduced phase separation method for removing $\mathrm{MgSO}_{4}{ }^{10}$. A basic challenge within the preparation of nanocomposites membrane is the homogeneity within the mixing between the nanoparticles and the organic component, which is resolved by using a silane coupling agent as a surface modifier for the SNPs ${ }^{22}$.

In this work, we investigated the fabrication of a CA composite membrane by the dry-wet phase inversion technique and studying the effect of SNPs addition prepared by sol-gel method on the 
characteristic and performance of RO CA membranes will be carried out. The role of 3aminopropyl triethoxysilane (APTES) on the modified SNPs as an additive for CA membrane with different ratios will be compared with pristine SNPs. The performance of the nanocomposite CA membranes represented in salt rejection and permeate water flux will be evaluated using brackish water of 10,000 ppm sodium chloride.

\section{Materials and methods}

Cellulose acetate was obtained from Across Organics Company with 39.8\% acetyl content and its average number molecular weight was 100,000 Da. Acetone was purchased from Fisher Scientific Company. Dioxane was purchased from Cambrian Chemical Company. Acetic acid and methanol were purchased from Carlo Erba reagents SAS Inc. Ammonium hydroxide (25\%) was purchased from Gateway Co. Ethanol was purchased from Carlo Erba reagent SPA Inc. (3-Aminopropyl) triethoxisilane (APTES) was obtained from Across Organic Company. Trimethyl orthosilicate (TMOS) was bought from Across Organics Company. Sodium chloride was supplied from MP Biomedicals, LLC, France.

\subsection{Preparation of SNPs}

A solution mixture of ethanol $(50 \mathrm{~mL})$, water $(1 \mathrm{~mL})$, and aqueous ammonia $(2.7 \mathrm{~mL})$ were added to a flask. An amount of TMOS $(1.5 \mathrm{~mL})$ was rapidly added to the mixture solution and magnetically stirred $(200 \mathrm{rpm})$ for $3 \mathrm{hrs}$. at $40^{\circ} \mathrm{C}$ under reflux. Then TMOS $(1 \mathrm{~mL})$ was dropwise added into the above solution and kept stir for another $3 \mathrm{hrs}$. The SNPs were harvested by centrifugation at $9000 \mathrm{rpm}$ for 10 minutes and washed twice with copious ethanol, and dried in an oven at $70^{\circ} \mathrm{C}$ for $24 \mathrm{~h}$.

\subsection{Preparation of MSNPs}

An amount of the prepared SNPs $(0.1 \mathrm{~g})$ were dispersed in ethanol $(20 \mathrm{~mL})$, and the solution was heated to $80^{\circ} \mathrm{C}$ under reflux. Then APTES $(1 \mathrm{~mL})$ was added to the solution. The mixture was boiled under reflux for $6 \mathrm{hrs}$, and followed by centrifugation at $9000 \mathrm{rpm}$ for 10 minutes, washed with ethanol several times, and dried in an oven at $70^{\circ} \mathrm{C}$ for $24 \mathrm{hr}$.

\subsection{Preparation of CA-RO nanocomposite membranes}

CA-RO control membrane was prepared as reported by Ali et al ${ }^{23}$. SNPs and MSNPs with different ratios $(0,10,30,50$, and $70 \mathrm{mg})$ were added to the solvent mixture and sonicated for 2 hrs to ensure complete dispersion before adding the CA powder. The nanocomposite membranes were denoted by the names S1, S3, S5, and S7 according to the concentration of SNPs of 10., 30, 50 , and $70 \mathrm{mg}$ respectively. The MSNPs nanocomposite membranes were denoted the names MS1, MS3, MS5, and MS7 according to the same concentration of MSNPs. 


\subsection{Characterization techniques}

The functional groups of the SNPs, MSNPs, and the prepared membranes were analyzed using Fourier Transform Infrared Spectroscopy (FTIR Spectrum BX 11 spectrometer FTIR LX 18-5255 Perkin Elmer). The surface morphology and cross-sectional images of the obtained membranes were studied using scanning electron microscopy (Joel JSM 5300, Japan EM). Samples were prepared as follows. The dried membranes were cut under liquid nitrogen and mounted on a brass plate. The membranes were then sputter-coated with a thin film of gold. Morphology and particle size were investigated using transmission electron microscopy (TEM) (JEOL, JEM-100CX). A small portion of SNPs and MSNPs powder was dispersed in ethanol. The solution undergoes ultrasonication for 10 minutes then we added one drop of this solution to the $\mathrm{Cu}$ grid to be analyzed. The hydrophilicity measurements of the surface of the membrane were measured using Rame-Hart, Instrument Company, France. A drop of distilled water of about $2 \mu \mathrm{L}$ was placed on the membrane surface $(3 \mathrm{~cm} \times 2 \mathrm{~cm})$ using a microsyringe (Hamilton Company, Reno, NV). The contact angle was measured within $20 \mathrm{sec}$ after the water drop was placed. To improve the statistics of the contact angle measurement, each reported data point is the average of five measurements at different positions at the same condition. X-ray diffraction analysis were obtained using (X-ray 7000 Shimadzu-Japan) at room temperature. The Bragg angle has $2 \theta$ in the range from 10 to 80 degrees to determine the degree of crystallinity of the prepared SNPs and MSNPs. The X-ray source is $\mathrm{Cu}$ target generated at $30 \mathrm{kV}$ and $30 \mathrm{~mA}$ with scan speed $4 \mathrm{deg} / \mathrm{min}$.

\subsection{Lab-scale RO performance test}

Performance measurements represented in the salt rejection and water flux were carried out by a cross-flow RO unit which consists of a commercially available crossflow stainless-steel CF042 cell with a membrane area of $42 \mathrm{~cm}^{2}$, a hydraulic pump of maximum pressure 60 bar, with a pressure control valve and a gauge through rejection line. A flow meter F-550 (USA) was connected to obtain a constant flow of 1 liter/min. The membranes were flushed in the RO unit system with deionized water for 30 min until a steady permeate flux was achieved, then the saline water with an initial feed concentration of $10,000 \mathrm{ppm} \mathrm{NaCl}$ was introduced. The $\mathrm{pH}$ and the temperature of the feed solution were kept constant at 7 and $25^{\circ} \mathrm{C}$, respectively. The salt concentrations were determined using a TDS meter (430 portable, Jenway, England). The permeate water flux was calculated using the following equation ${ }^{23}$ :

$$
\text { Permeate water flux }=\frac{Q}{A . t}
$$

where $\mathrm{Q}$ is the quantity of permeate $(\mathrm{L}), \mathrm{A}$ is the effective membrane area $\left(\mathrm{m}^{2}\right)$ and $\mathrm{t}$ is the time (hr).

The percentage of the salt rejection (SR) is calculated using the following equation ${ }^{23}$ : 


$$
\text { Salt rejection }=\frac{C f-C p}{C f} 100 \%
$$

where $C f$ is the salt concentration of the feed solution and $C p$ is the salt concentration of the permeate water.

\section{Results and discussion}

\subsection{SNPs and MSNPs structure and crystallinity}

The chemical structures of the SNPs and the MSNPs are analyzed using FTIR as displayed in Figure 1(a). The band around $3414 \mathrm{~cm}^{-1}$ is assigned to the $-\mathrm{OH}$ stretching vibrations of the silanol $\mathrm{Si}-\mathrm{OH}$ groups or the adsorbed water molecules on the silica surface. A sharp peak at $1633 \mathrm{~cm}^{-1}$ is found as the scissor bending vibrations of the trapped water molecules in the silica. The strong band at $1105 \mathrm{~cm}^{-1}$ coincides with the asymmetric vibrations of the $\mathrm{Si}-\mathrm{O}-\mathrm{Si}$ bond. The weak band at $941 \mathrm{~cm}^{-1}$ signifies the $\mathrm{Si}-\mathrm{O}$ asymmetric stretching vibrations of the silanol groups ( $\mathrm{Si}-\mathrm{OH}$ ). An absorption moderate peak at $790 \mathrm{~cm}^{-1}$ is appointed to the stretching vibrations of the $\mathrm{Si}-\mathrm{O}-\mathrm{Si}$ bond. The band noticed at $455 \mathrm{~cm}^{-1}$ is owing to the bending vibrations of the $\mathrm{Si}-\mathrm{O}-\mathrm{Si}$ bond ${ }^{24-28}$. The characteristic bands of the MSNPs have the same as in the spectrum of SNPs. There is a peak at $3373 \mathrm{~cm}^{-1}$ caused by the existence of NH vibrations stretching results from ATES. Furthermore, a peak has emerged at $2914.5 \mathrm{~cm}^{-1}$ is attributed to $\mathrm{CH}_{2}$ vibrations (stretching). Additionally, at 1404 $\mathrm{cm}^{-1}$ there is a peak recognized to $\mathrm{CH}_{3}$ bending vibrations ${ }^{29}$. The characteristics bands of silica become weak after modification, which confirms that the silane molecules have been effectively grafted onto the SNPs surface ${ }^{30}$.

Figure 1 (b) shows XRD patterns of SNPs and MSNPs. The broadband appears at Bragg angle $2 \theta$ of $24^{\circ}$ which is associated with amorphous silica. All forms of silica have a short range of order solids composed of silicon and oxygen atoms. $\mathrm{SiO}_{2}$ is a silicon oxide made up of linear triatomic molecules in which a silicon atom is covalently bonded to two oxygens. The $\mathrm{Si}$ atom shows tetrahedral coordination with four $\mathrm{O}_{2}$ atoms surrounding a central $\mathrm{Si}$ atom and consequently the SNPs forms three-dimensional networks in which each $\mathrm{Si}$ atom is covalently bonded in a tetrahedral manner to 4 oxygen atoms. The absence of any sharped peaks confirms the amorphous structure of silica $24,26,30,31$.

\subsection{Morphological study of SNPs and MSNPs}

The microstructure of the SNPs and the MSNPs are examined in Figure 1(c, d) by TEM. The SNPs have a uniform, homogeneous spherical structure with a controlled average particle size of 26-51 $\mathrm{nm}$. On the other hand, the MSNPs are slightly irregular in shape and seem approximately spherical. The diameter of these nanoparticles is estimated to be about 26.7-29.1 nm. The agglomeration shown in the figure may be due to the inhomogeneous surface functionalization via the post-synthetic grafting method as has been explained by Gu et al ${ }^{32}$. 

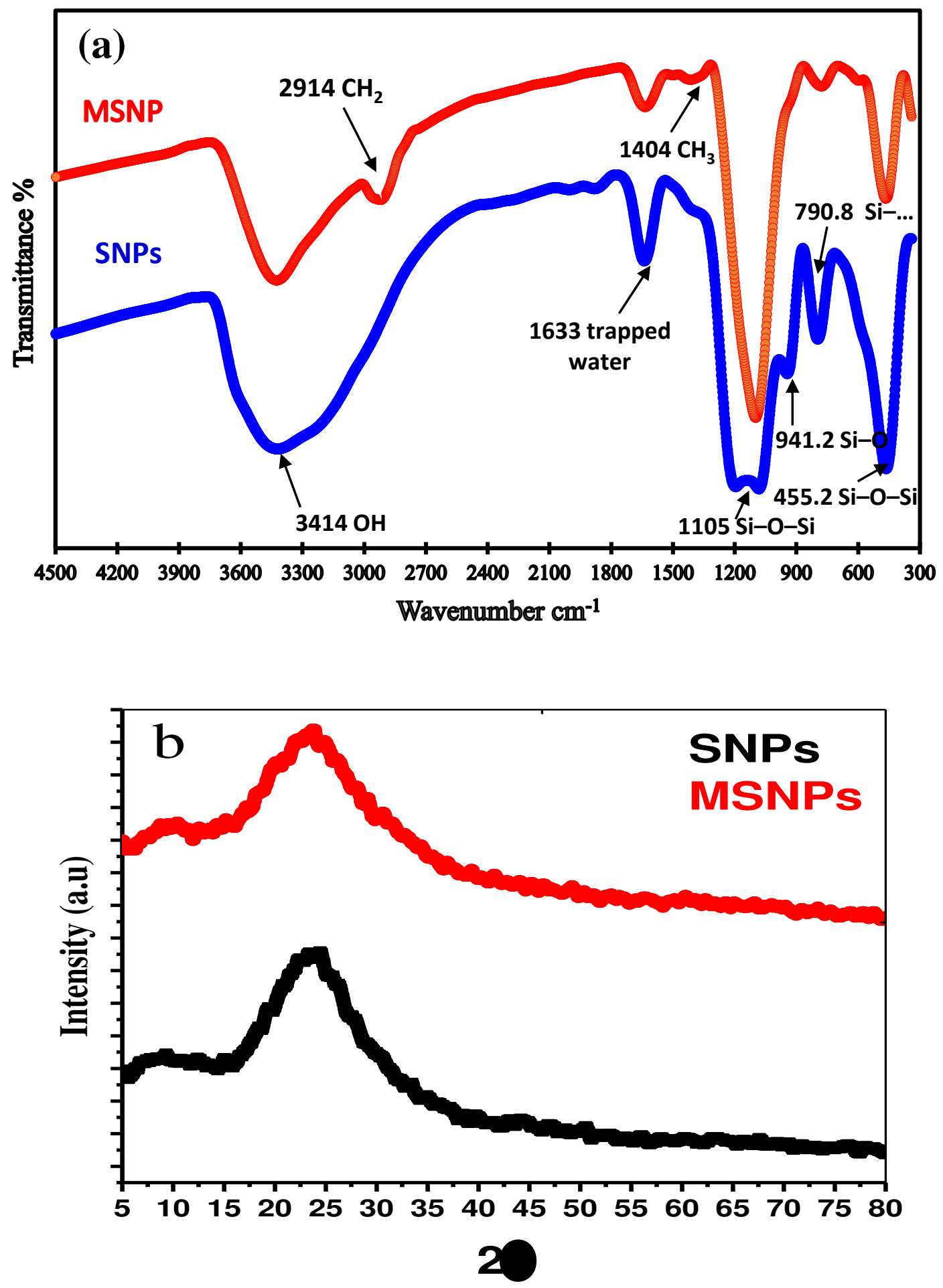

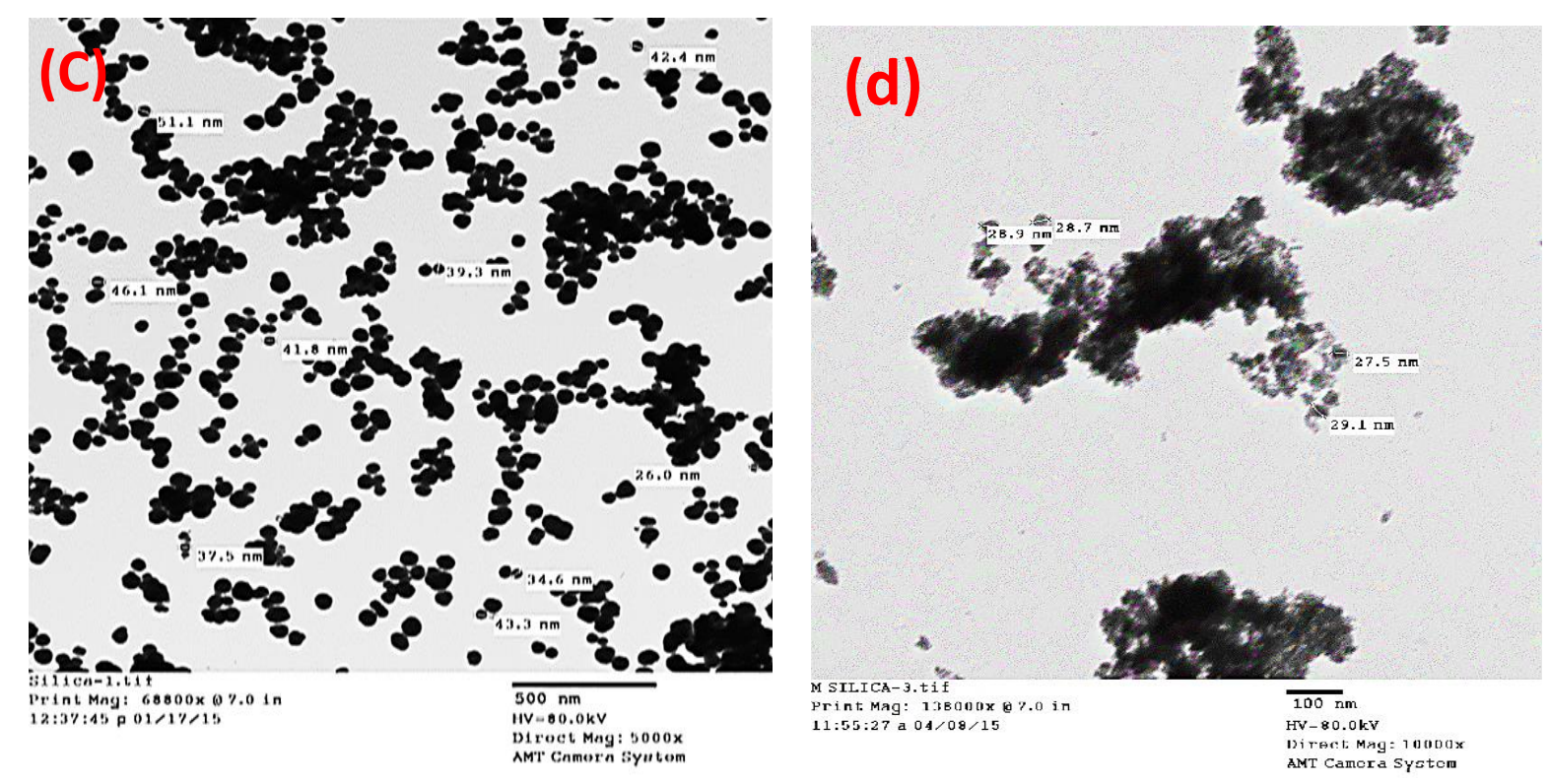

Figure (1) Characterization of SNPs and MSNPs. FTIR spectra (a), XRD patterns (b), TEM images (c, d).

\subsection{Nanocomposite membranes properties and their desalination performance}

\subsubsection{Chemical structure of nanocomposite membranes}

As shown in Figure 2 (a), for the control CA and nanocomposite membranes based on SNPs there is broadband at $3484 \mathrm{~cm}^{-1}$ and a peak at $1372 \mathrm{~cm}^{-1}$ which are designated for stretching and bending vibration of the $\mathrm{OH}$, respectively. The peak at $2940 \mathrm{~cm}^{-1}$ is characteristic of the aliphatic C-H group and the stretching vibration of the $\mathrm{C}=\mathrm{O}$ bond is observed around $1748 \mathrm{~cm}^{-1}$. The peaks at $1636 \mathrm{~cm}^{-1}$ and $1512 \mathrm{~cm}^{-1}$ represent the free water molecule and the stretching mode of the $\mathrm{C}=\mathrm{C}$ bond, respectively. The bending vibration of $\mathrm{CH}_{2}$ is observed at $1434 \mathrm{~cm}^{-1}$, the band at $1234 \mathrm{~cm}^{-1}$ is attributed to the stretching vibration of the $\mathrm{C}-\mathrm{O}$ group, the absorption band at $1054 \mathrm{~cm}^{-1}$ is corresponded to the ether linkage C-O-C from the glycoside unit, and the peak at $908 \mathrm{~cm}^{-1}$ is assigned to the (pyranose ring) out-of-plane $\mathrm{C}-\mathrm{H}$ bend ${ }^{33-37}$. It is noticed that the intensity of the carbonyl band of the CA control membrane has decreased with the addition of SNPs in the composite membrane, which confirmed the development of hydrogen bonding between the carbonyl and the hydroxyl groups. There is a slight shift from $1748 \mathrm{~cm}^{-1}$ for the CA control membrane to $1743.8 \mathrm{~cm}^{-1}$ for $\mathrm{S} 7$, which confirmed the carbonyl group interaction through hydrogen bonding with SNPs. These results indicate the interactions of SNPs in CA nanocomposite membranes ${ }^{33}$. 
Figure 2(b) illustrates the impact of various concentrations of MSNPs on the CA membrane. It is noticed that there are two overlapping bands that occurred with an increase in the intensity of the peaks and the peak at $3484 \mathrm{~cm}^{-1}$ is assigned to the $\mathrm{OH}$ group in the $\mathrm{CA}$ control and $\mathrm{NH}_{2}$ group for the MSNPs. The other overlapping at $1636 \mathrm{~cm}^{-1}$ is assigned to the water of crystallization in the CA membrane and the $\mathrm{NH}_{2}$ group in the modified MSNPs. ${ }^{33-36,38}$. These results prove that the CA membrane has been successfully modified with the $\mathrm{NH}_{2}$ group.

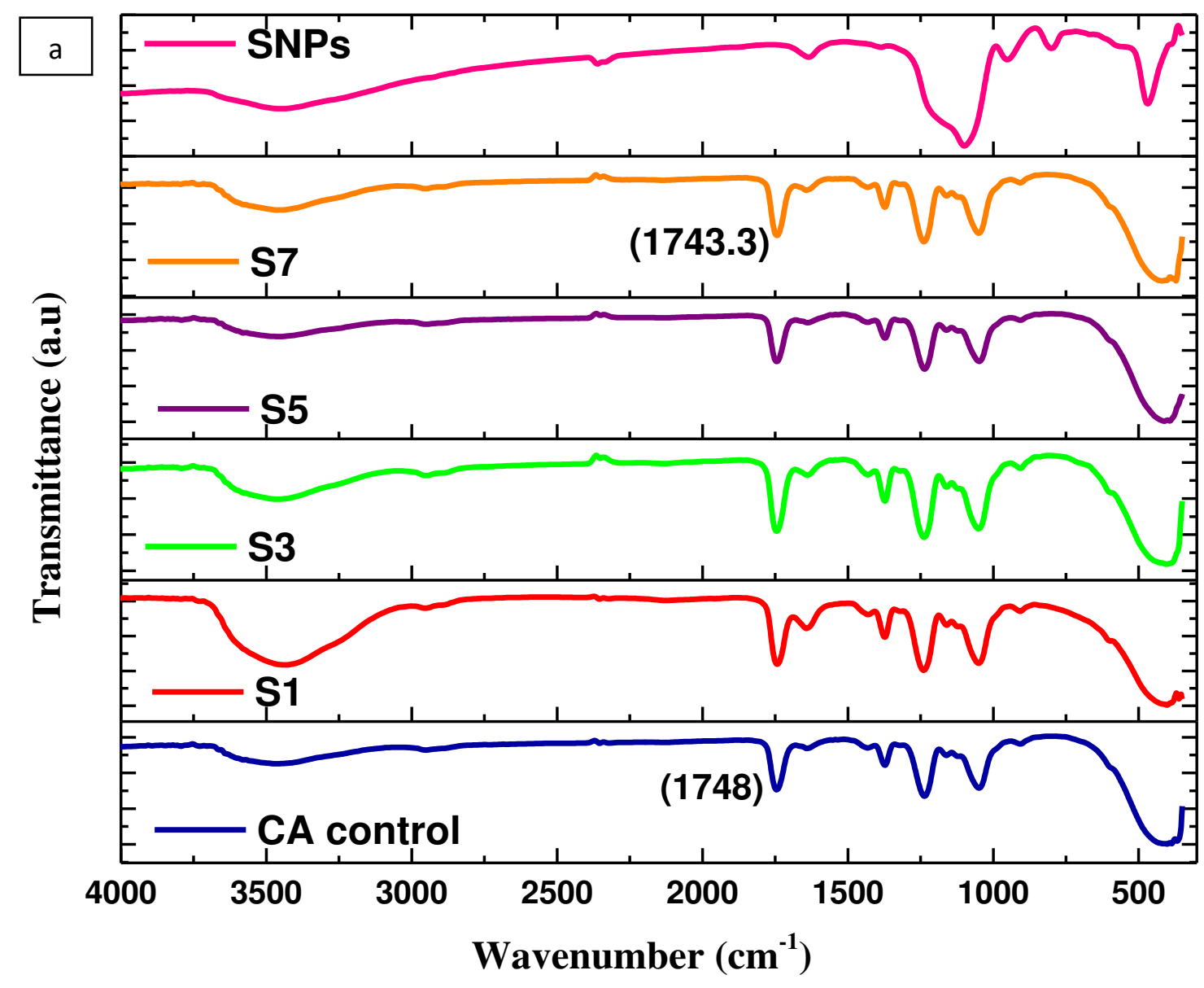




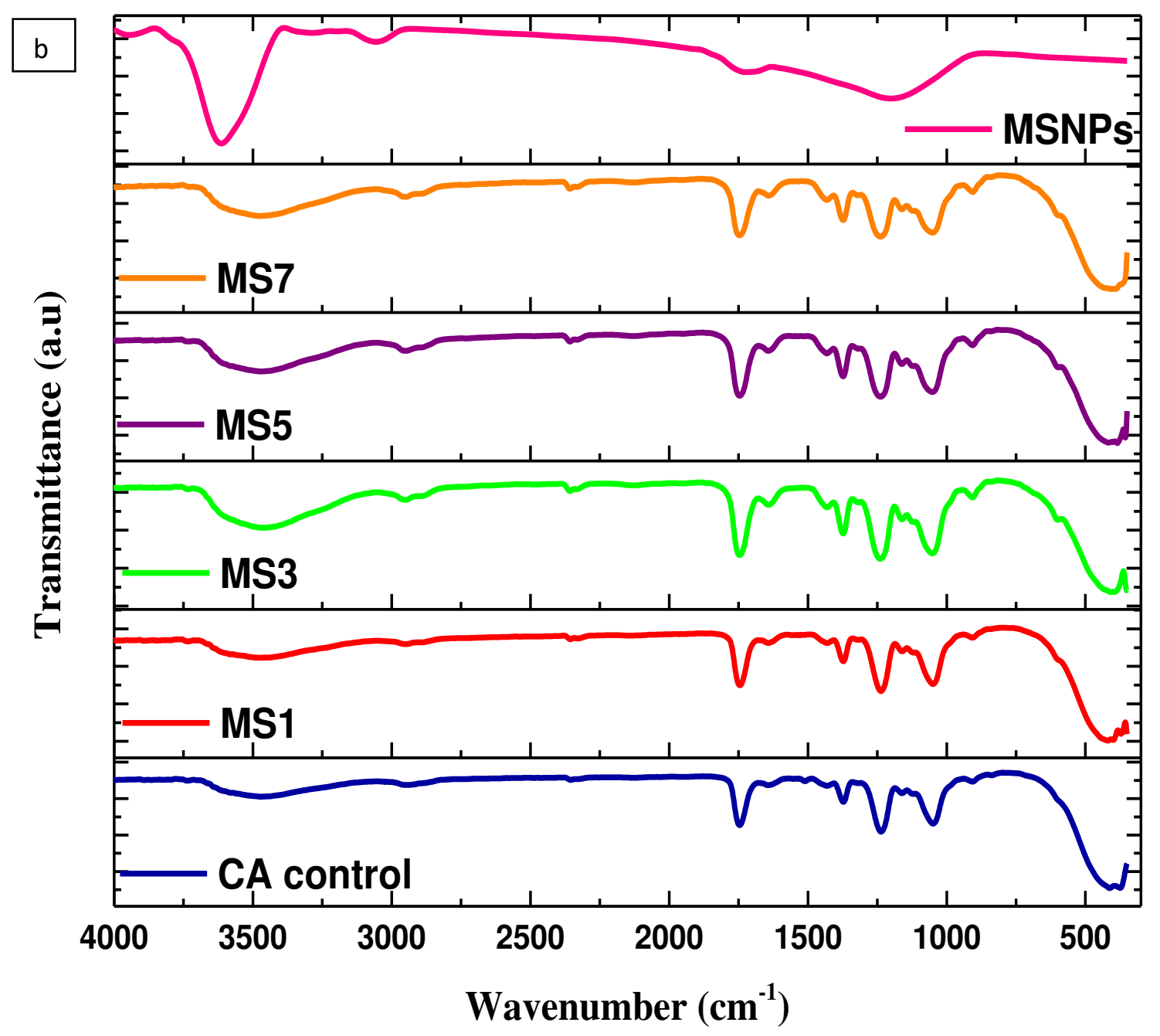

Figure 2: FT-IR spectra of CA nanocomposite membranes: Different concentrations of SNPs (a), Different concentrations of MSNPs (b).

\subsubsection{Morphology of CA nanocomposite membranes}

The addition of SNPs and MSNPs to the CA membrane has a great effect on the membrane morphology and structure. The SEM images in Figure 3 depict distinct changes in the morphology of the CA control membrane and the CA-SNPs nanocomposite membranes concerning the surface layer, cross-section, and the bottom of the membranes as a result of varying SNPs concentrations. All the surfaces of the membranes, including the CA control membrane, show a ridge and valley 
structure. Notably, the cross-section structure of the CA control and the nanocomposite membranes reveals fingers within sponge structure, Nonetheless, the nanocomposite membranes fingers have a different form than the control membrane. Due to the hydrophilic nature of SNPs, the solvent-nonsolvent exchange in the phase inversion process is accelerated and formed a dense skin layer ${ }^{18}$. In the case of the S3 membrane, more voids are formed with respect to CA control membrane and S1 membrane ${ }^{39}$. Further increase of the SNPs concentrations from S5 to S7, the polymer solution viscosity is slightly increased, causing delayed demixing and agglomeration of SNPs with lower voids in the cross-section structure. This agglomeration also forms pores in the bottom surfaces. The formed dense skin layer limits the exchange of the solvent and nonsolvent in the nanocomposite and thus, suppresses the pore formation in the membranes ${ }^{18,40-42}$. From the measurements of the membranes' dense layer thickness, it can be concluded that, as the concentration of SNPs increases, the dense layer thickness increases. The dense layers thickness of the CA-SNPs nanocomposite membranes is $1.18,1.43,1.62,1.72$, and $1.81 \mu \mathrm{m}$ for CA control, S1, S3, S5, and S7 membrane, respectively.

The SEM images in Figure 4 display distinct changes in the morphology between the CA control membrane and the CA-MSNPs nanocomposite membranes. All the nanocomposite membranes exhibit ridge and valley surfaces. Increasing the MSNPs concentration in the CA membranes causes some agglomeration of MSNPs which results in relatively delayed demixing in nanocomposite membranes with a high concentration of MSNPs $(50$ and $70 \mathrm{mg}$ ) in comparison with a lower concentration of MSNPs (10 and $30 \mathrm{mg}$ ) in the nanocomposite membranes. Thus, the formation of macrovoids in the membrane matrix is suppressed ${ }^{40-42}$. The membranes' surface tends to be smoother, and the surface roughness decreases as the concentration of MSNPs increases. The bottom of the CA control membrane is a smooth and flat structure while the modified membranes tend to be smoother, and fewer bends are observed. All CA-MSNPs nanocomposite membranes exhibited a fingers-like structure in the cross-section, separated by thick walls of sponge structure, except MS1 which showed a distinct tear structure shape. It is obvious that as the concentration of the MSNPs increases the dense thin layer of the membrane increases. The dense layer is 1.18, 1.3, $1.56,1.66$, and 1.88 for CA control, MS1, MS3, MS5, and MS7 membrane, respectively. 


\section{Surface}

CA control

S1

S3

S5

S7
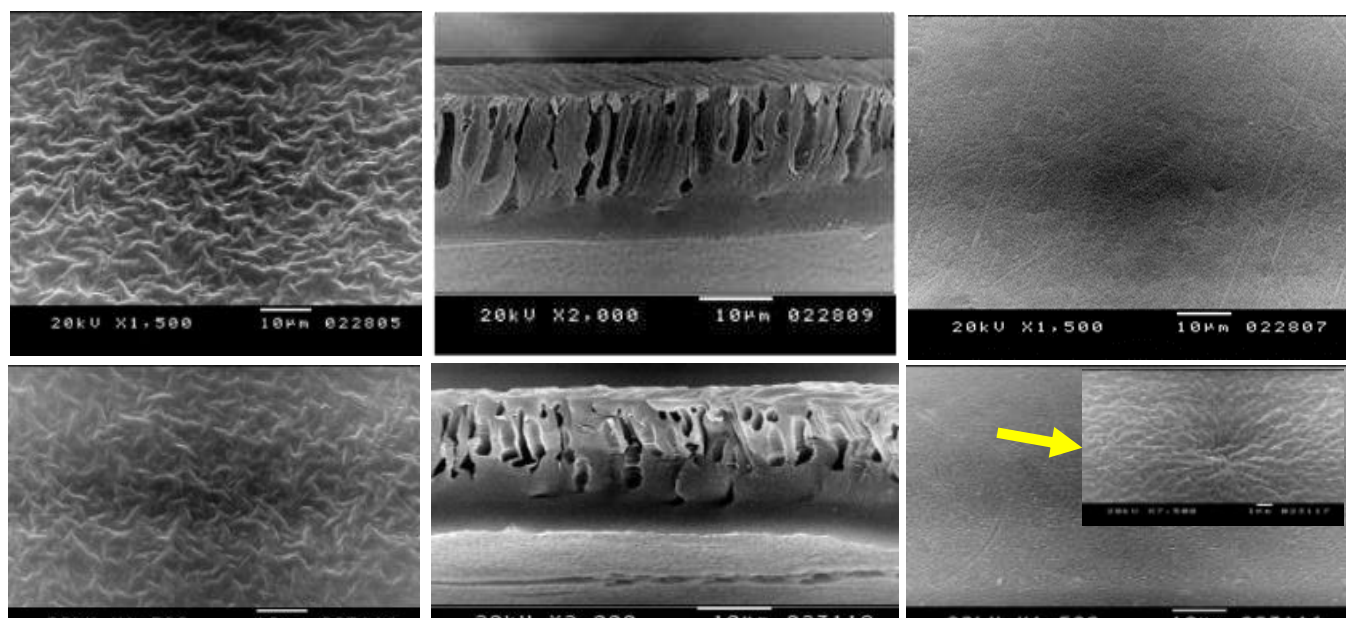

$20 \mathrm{kU} \times \mathrm{x}=500$
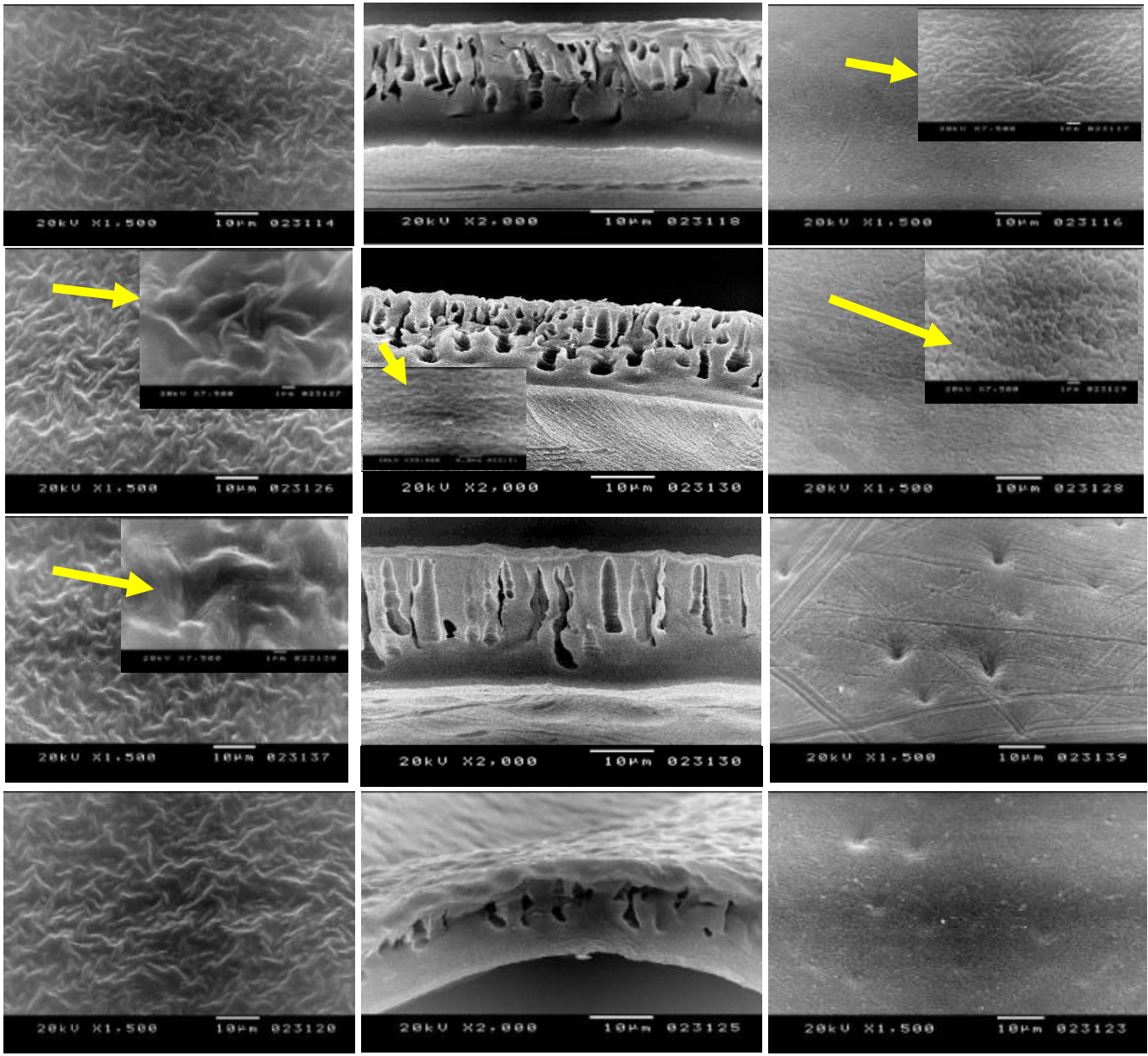

Figure 3: SEM images of control CA and CA-SNPs nanocomposite membranes with different ratios. 
Surface

Cross-section
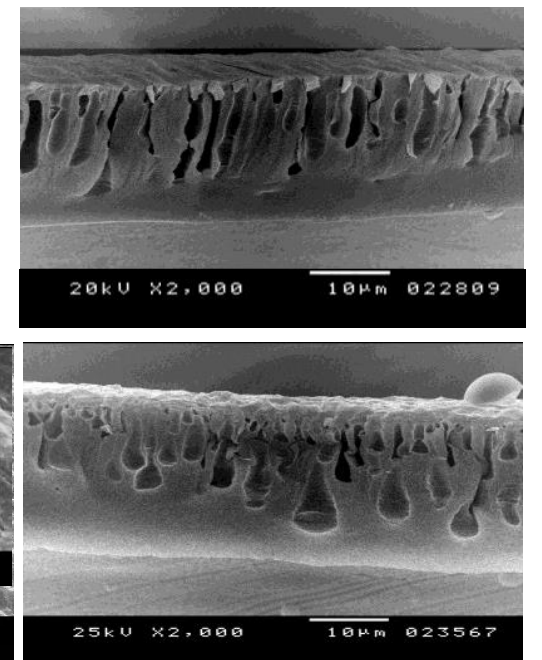

MS1

MS3
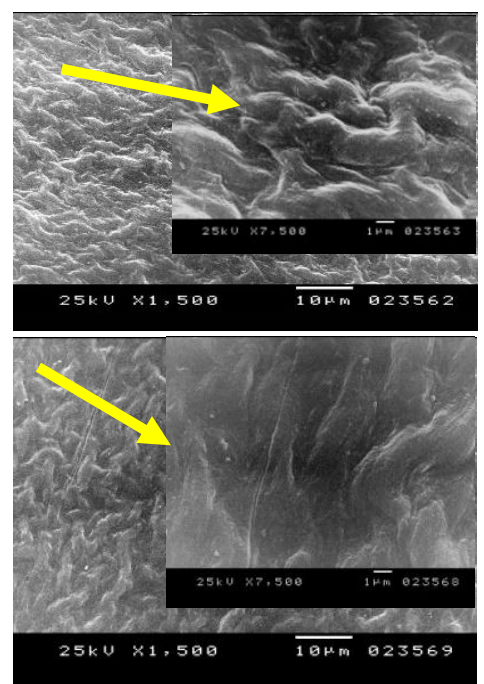

MS5

MS7

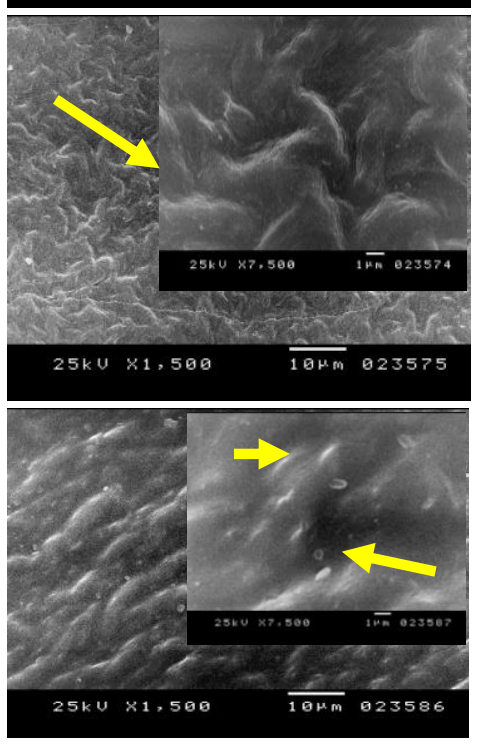

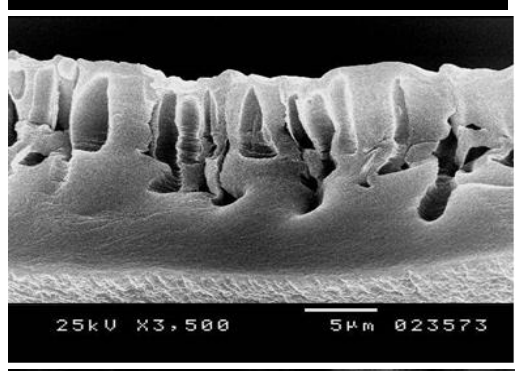
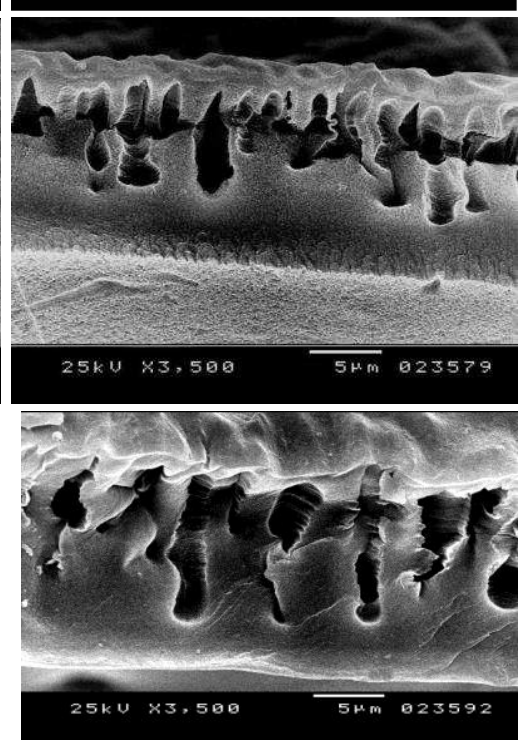

Bottom
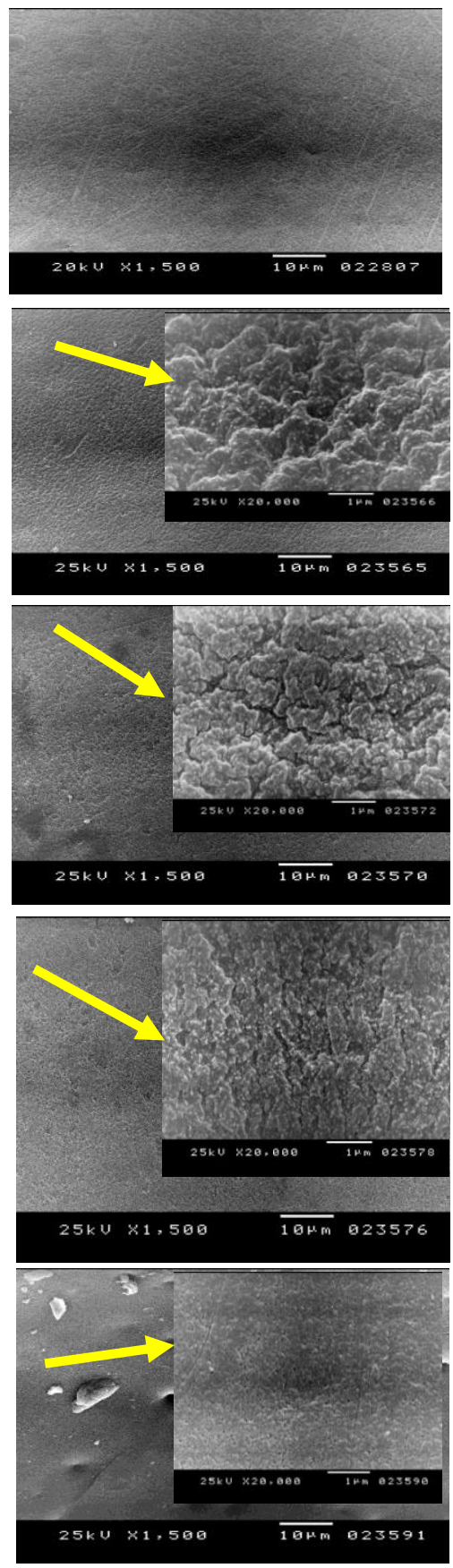

Figure 4: SEM images of control CA and CA- MSNPs nanocomposite membranes with different ratios. 


\subsubsection{Membranes' hydrophilicity}

Figure 5 ( $a$ and $b$ ) manifests the relationship between SNPs and MSNPs concentrations in the CA nanocomposites and their water contact angle measurements. As the SNPs concentration in the CA nanocomposite membrane increases, the measured water contact angle is slightly increased. This is maybe due to their agglomeration on the membrane surface. The water contact angle of the control CA is 54.5 degrees, while the water contact angle measurements for the CA-SNPs nanocomposite membranes are ranged from 66.2 to 56.6 degrees. However, for the CA- MSNPs nanocomposite membranes, they maintain their hydrophilic nature compared with the CA control membrane. Generally, the values of water contact angle tend to slightly decrease from (MS1 to MS5) by increasing the MSNPs concentration in the CA nanocomposite membranes. MS5 has a water contact angle of 52.28 degrees. This is the least water contact angle obtained for the CAMSNPs nanocomposite membrane. This is due to the hydrophilic amino groups attached to the silica surface ${ }^{43}$. Then, increasing the concentration of MSNPs to $70 \mathrm{mg}$ causes an increase in the water contact angle (57.42 degrees). This increase in water contact angle measurement is attributed to the agglomeration of MSNPs.

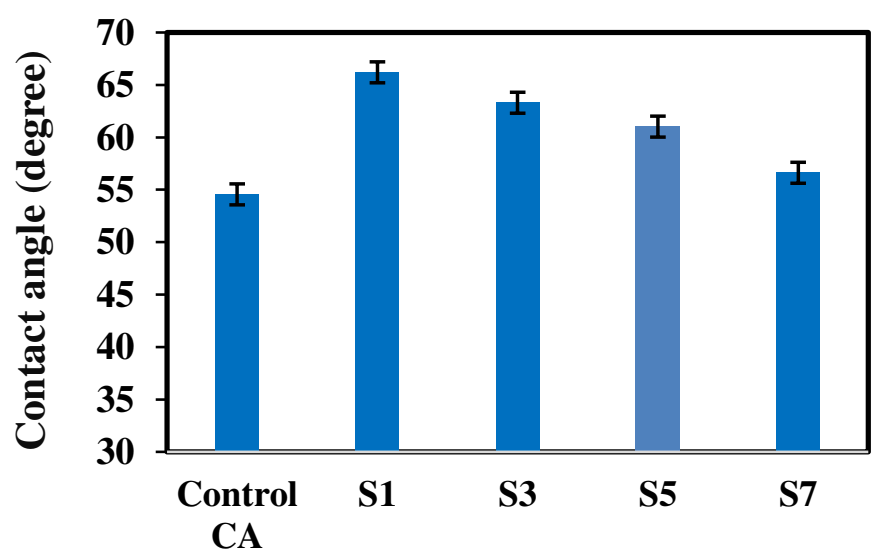

(a)

Membrane type

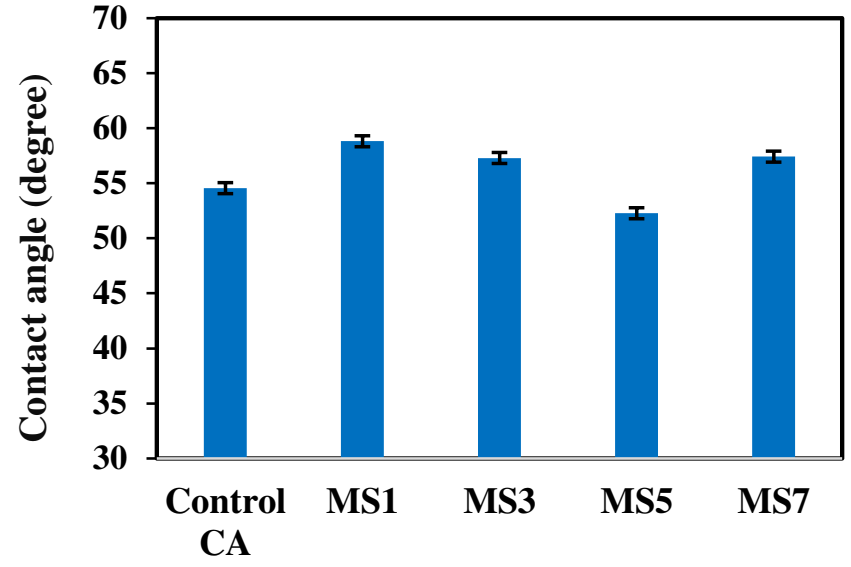

(b)

Membrane type

Figure 5: Water contact angles of different SNPs (a), MSNPs (b) on CA membranes.

\subsubsection{The membranes' performance}

Figure 6 ( $a$ and $b$ ) presents the influence of SNPs concentrations on nanocomposite membranes salt rejection and permeate water flux for $10,000 \mathrm{ppm} \mathrm{NaCl}$ feed under different operating pressures from 8 to 50 bar. All the membranes salt rejection is decreasing with escalating pressure, and this is expected due to the concentration polarization phenomena. For all nanocomposite membranes, they show a sudden decrease in salt rejection compared to the CA control membrane which shows a gradual decrease in salt rejection. The sudden decrease is may be attributed to the generation of small nonselective voids existing between SNPs and the CA polymer chain ${ }^{25}$. The CA control 
membrane has $96 \%$ salt rejection at 10 bar and declined to $85 \%$ during the operating pressure between 14 and 25 bar, then it is ruptured at 28 bar. For S1, S3, and S5 the salt rejections decreased with increasing the pressure and could not tolerate the pressure of more than 20 bar. However, S7 indicates a decrease in rejection between 10 and 18 bar to reach $64 \%$ of salt rejection. Then the salt rejection increases again from 20 bar to about 35 bar and it appears a plateau region of stable salt rejection behavior up to 48 bar then it is ruptured at 50 bar. This is due to the densification of the dense skin layer on S7 surface ${ }^{44}$. This trend can be explained based on that the addition of SNPs causes a formation of the thicker skin layer and raises the viscosity of the polymer and SNPs solution. This results in the suppression of microvoids, and the formation of a dense layer of the S7 membrane ${ }^{18}$.

For all membranes, as indicated by Figure 6 (b), the permeate water flux has increased with the increasing of the operating pressure. This is due to the increasing trans-membrane pressure, which significantly enhances the pure permeate water flux during the period of operation. The highest permeate water flux obtained is $60 \mathrm{~L} / \mathrm{m}^{2}$.h at 50 bar for the $\mathrm{S} 7$ membrane. Although $\mathrm{S} 7 \mathrm{has}$ a rejection less than the CA control membrane it shows a better permeate water flux throughout the operation. At 10 bar, as a reference pressure for all the membranes, $\mathrm{S} 1$ has a permeate water flux of $2.6 \mathrm{~L} / \mathrm{m}^{2} . h$ compared to the CA control membrane which has $1 \mathrm{~L} / \mathrm{m}^{2}$.h. However, S1 shows a decrease in rejection compared to the CA control by only $1 \%$. On the other hand, during the operating process and increasing the SNPs in the nanocomposite membrane an enhancement of the permeate water flux until it reached the maximum in a membrane in S7 is observed. This is due to the formation of voids in the S7 membrane that facilitated the water transport.

Figure 7 (a) shows the performance of the CA-MSNPs nanocomposite membranes in terms of salt rejection out of $10,000 \mathrm{ppm} \mathrm{NaCl}$ feed under different operating pressures from 8 to $35 \mathrm{bar}$. The salt rejection of all membranes decreases with the pressure because of the concentration polarization phenomena. It is noticed that the rejection of MS7 increases with the operating pressure and reaches $91.6 \%$ at 34 bar. This may be due to the dense skin layer compaction which affected the rejection of the nanocomposite membrane ${ }^{44}$. Also, the viscosity may have played a role here, the MS7 membrane tends to be dense because of the presence of a high concentration of MSNPs (70 mg), which will lead to increasing the viscosity of the solution and consequently this membrane tends to have a sponge structure with the formed dense skin layer. Under continuous operation and increasing pressure, the skin layer may tend to be compacted and more impermeable to salt penetration ${ }^{44}$.

Figure 7 (b) depicts the variation of permeate water flux of CA- MSNPs nanocomposite membranes out of $10,000 \mathrm{ppm} \mathrm{NaCl}$ feed under different operating pressures from 8 to 35 bar. For all the nanocomposite and control membrane, as the operating pressure increases the permeate water flux increases. This is due to the increasing impact of the driving force of the penetration of water. It is noticed that for the operating pressure that ranges from 10 to 20 bar, the permeate water flux of both MS1 and the CA control membrane is almost constant. Then the permeate water flux of the CA control membrane increases until it reaches its highest value at $26.18 \mathrm{~L} / \mathrm{m}^{2} . \mathrm{h}$ at 28 bar. While the permeate water flux of the MS1 membrane increases also with the operating pressure to reach its highest value at $3.8 \mathrm{~L} / \mathrm{m}^{2} . \mathrm{h}$ at 26 bar. The MS3 membrane shows the highest permeate water flux among other nanocomposite membranes with a value of $35.7 \mathrm{~L} / \mathrm{m}^{2} . \mathrm{h}$ at $24 \mathrm{bar}$. Then the permeate water flux decreases to $32.13 \mathrm{~L} / \mathrm{m}^{2} . \mathrm{hr}$ at 26 bar and this behavior is attributed to the membrane compaction. MS5 and MS7 have permeated water flux less than that of MS3 due to the 
agglomeration effect of the MSNPs in MS5 and MS7. The compaction of the membrane has a significant role where the polymer chains are forced to occupy a smaller total volume of space, under continuous and the increased pressure. In this case, the water penetration through the membrane is difficult and the salt permeability reduces as seen in MS7 ${ }^{44}$. For MS5 and MS7 membranes, the maximum permeate water flux values produced is $24.99 \mathrm{~L} / \mathrm{m}^{2} . \mathrm{h}$ at 22 bar and 5.33 $\mathrm{L} / \mathrm{m}^{2} . \mathrm{h}$ at 34 bar, respectively.

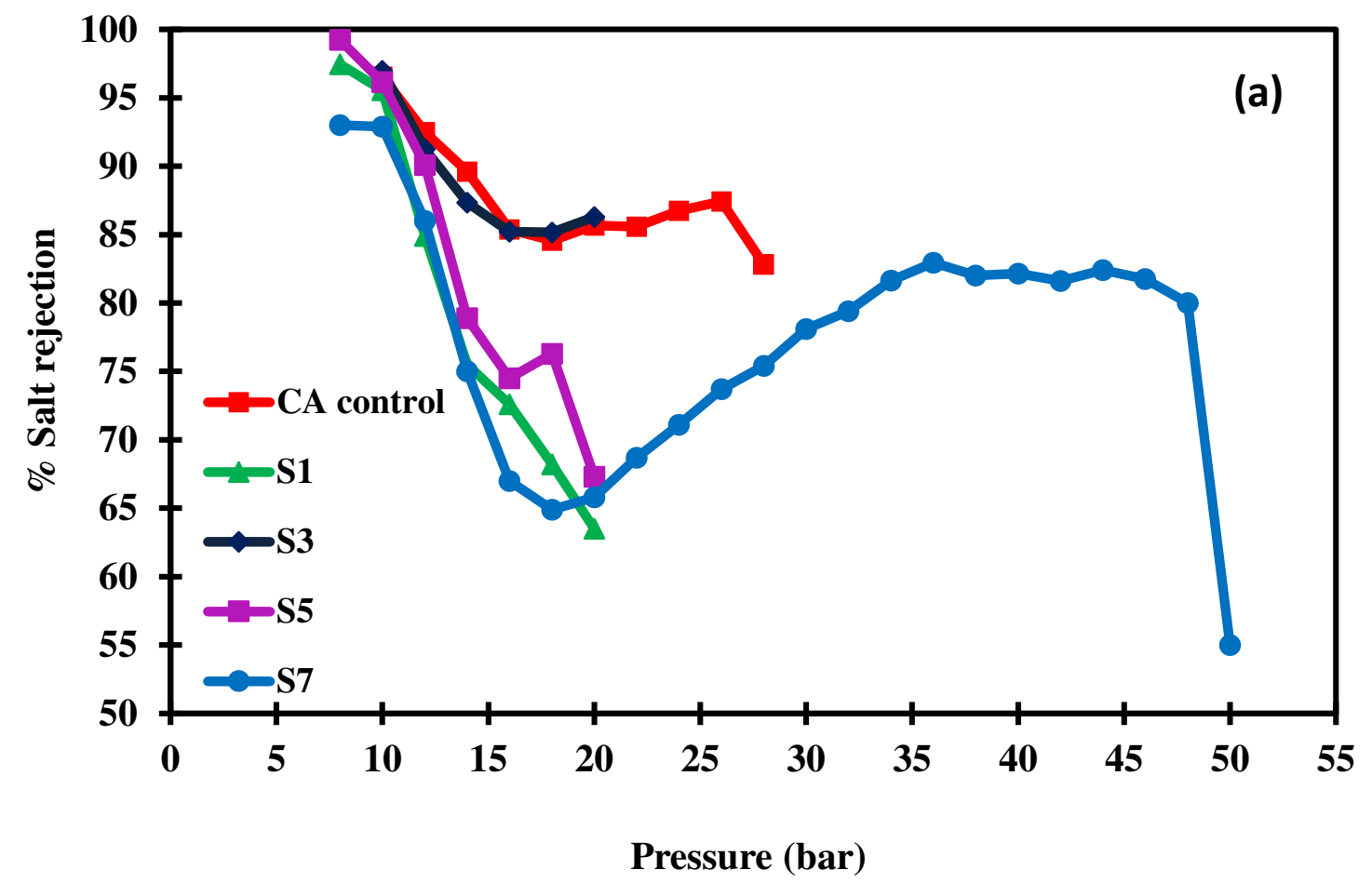




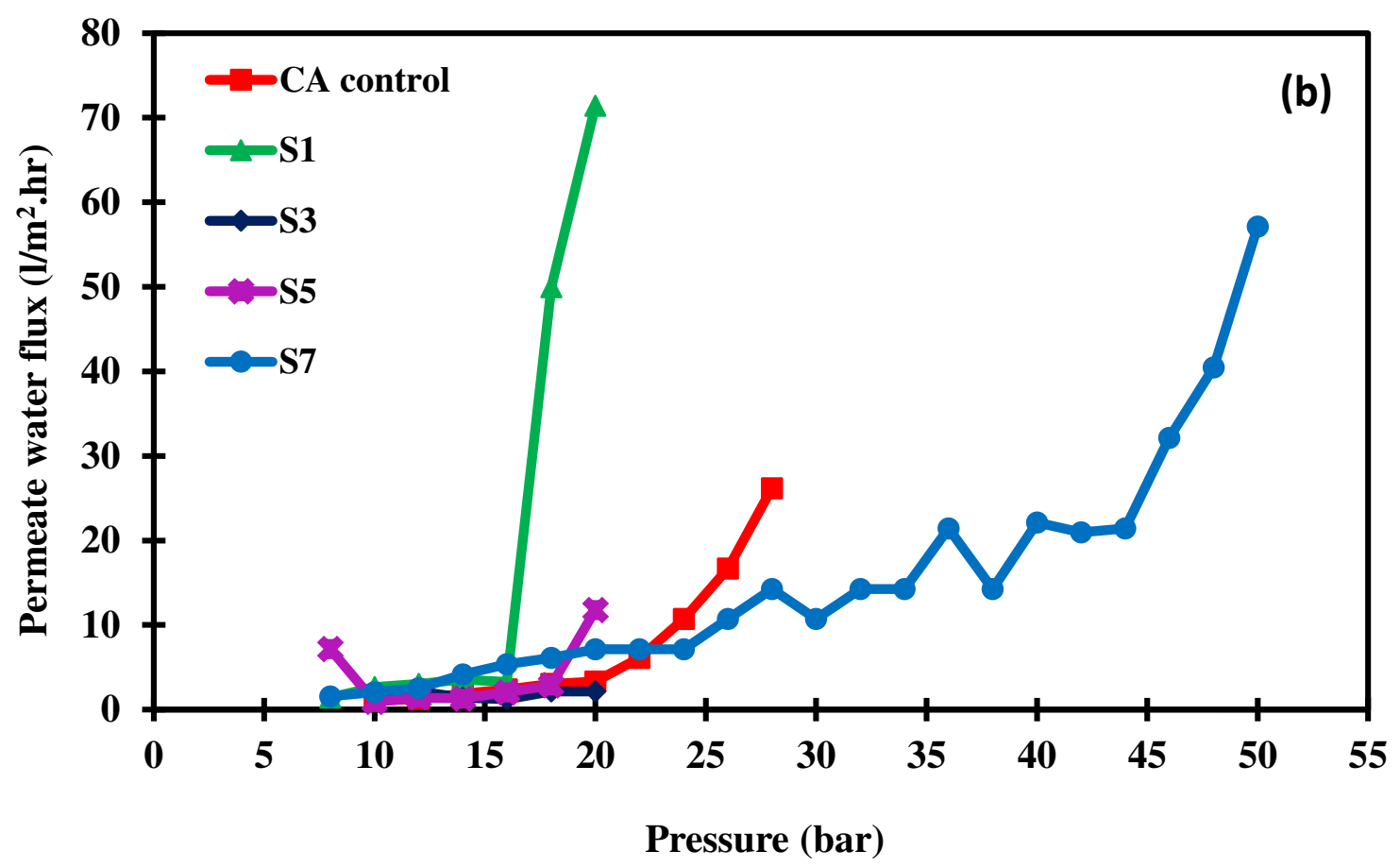

Figure 6: Salt rejection versus feed pressure of the membranes with different SNPs concentration (a). Permeate water flux versus feed pressure of membranes with different SNPs concentrations $(b)$.

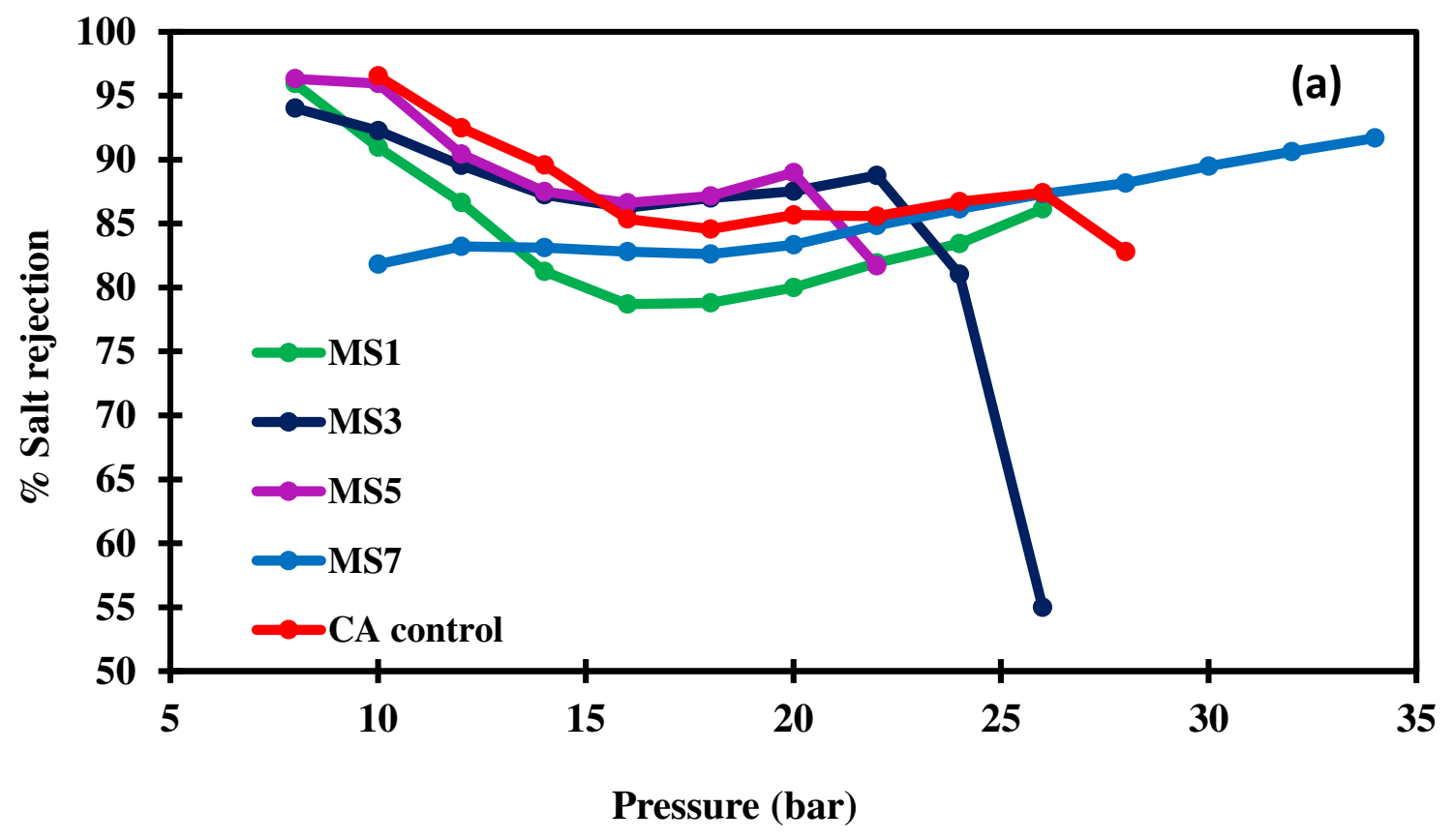




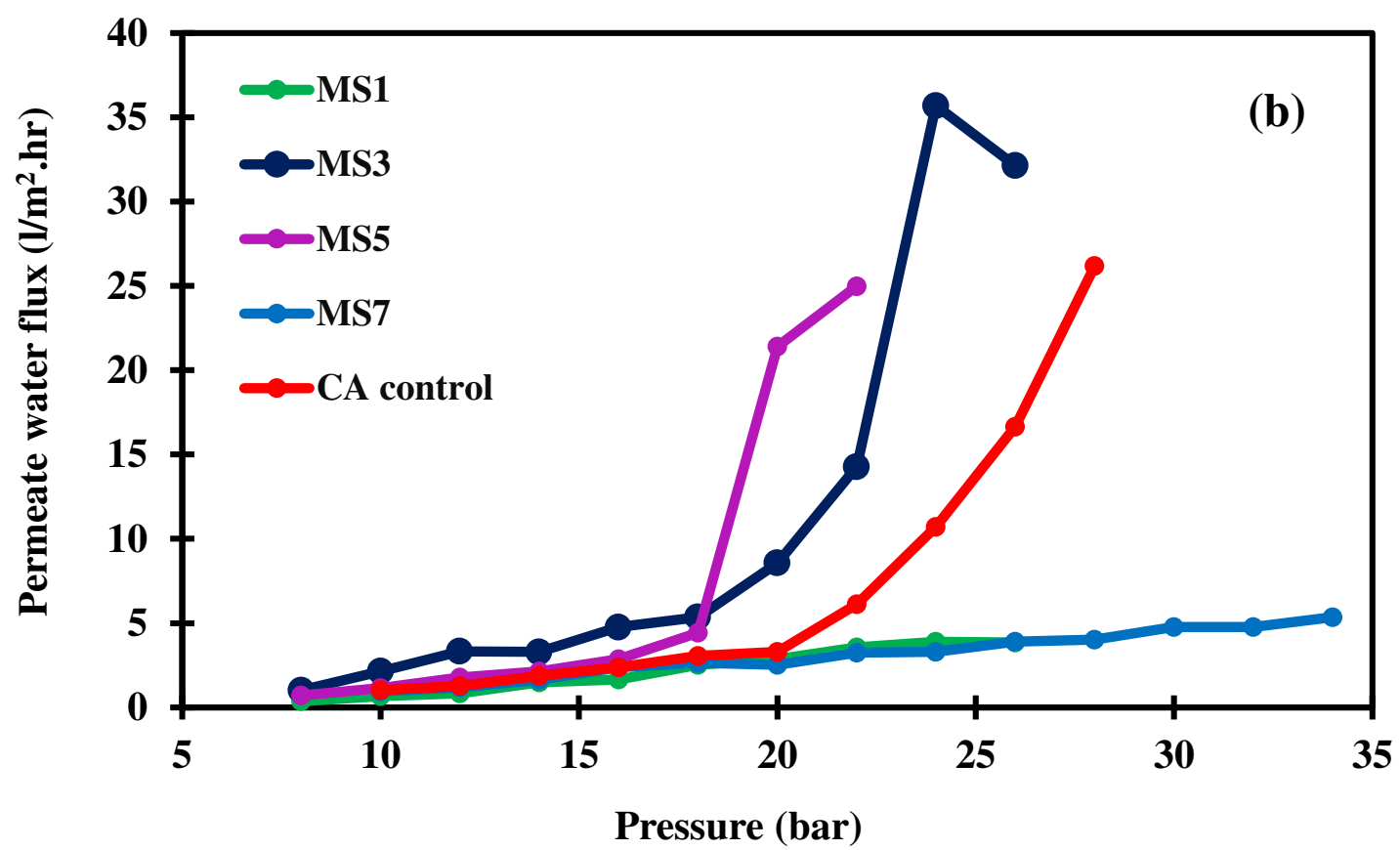

Figure 7: Salt rejection versus feed pressure of the membranes with different MSNPs concentration (a). Permeate water flux versus feed pressure of membranes with different MSNPs concentrations (b).

\section{Conclusion}

The properties of the nanoparticles and their preparation method have influenced the membrane properties and performance. SNPs prepared by the sol-gel method have influenced the CA membrane, the significant effect was the increase in permeate water flux of $1.6 \mathrm{~L} / \mathrm{m}^{2} . \mathrm{hr}$ more than the CA control membrane which had $11.6 \mathrm{~L} / \mathrm{m}^{2} . h r$. The MSNPs addition enhanced the CA membrane desalination performance due to the hydrophilic amino group on its surface, but the inhomogeneous surface functionalization via post-synthetic grafting as it was seen in the TEM image has led to the agglomeration of the MSNPs and hence affected the performance in that way. The addition of MSNPs enhanced the permeate water flux but there was a tradeoff in salt rejection. But the interesting thing for the addition of both SNPs and MSNPs at their concentration of $0.07 \%$ at both membranes they endured a high pressure unlike the CA control membrane which ruptured at $28 \mathrm{bar}$, they ruptured at $50 \mathrm{bar}$, hence at this concentration of SNPs and MSNPs the mechanical properties were enhanced.

\section{Declaration of interest statement}

The authors declare that this work has not been done or published before and no competing financial interest.

\section{Author contributions}

Amira S. Mohammed Ali and Marwa Khalil contributed to ideas, experiment execution, and interpretation of the data and writing of the manuscript. Moataz Soliman, Sherif Kandil, and Shaker Ebrahim supervised the experiments and edited the manuscript. 


\section{References}

1 Curto, D., Franzitta, V. \& Guercio, A. A Review of the Water Desalination Technologies. Applied Sciences 11, 670, https://doi.org/10.3390/app11020670 (2021).

2 Teow, Y. H. \& Mohammad, A. W. New generation nanomaterials for water desalination: A review. Desalination, https://doi.org/10.1016/j.desal.2017.11.041 (2017).

3 Anand, A., Unnikrishnan, B., Mao, J.-Y., Lin, H.-J. \& Huang, C.-C. Graphene-based nanofiltration membranes for improving salt rejection, water flux and antifouling-A review. Desalination 429, 119-133, https://doi.org/10.1016/j.desal.2017.12.012 (2018).

4 Basile, A., Cassano, A. \& Rastogi, N. K. Advances in membrane technologies for water treatment: materials, processes and applications. (Elsevier, 2015).

5 Idress, H. et al. Cellulose acetate based Complexation-NF membranes for the removal of $\mathrm{Pb}$ (II) from waste water. Scientific Reports 11, 1-14 (2021).

6 Baker, R. W. Membrane technology and applications. Membrane Technology (2004).

7 Warsinger, D. M. et al. A review of polymeric membranes and processes for potable water reuse. Progress in Polymer Science, https://doi.org/10.1016/j.progpolymsci.2018.01.004 (2018).

8 Ursino, C. et al. Progress of Nanocomposite Membranes for Water Treatment. Membranes 8, doi: 10.3390/membranes8020018 (2018).

9 Pendergast, M. M. \& Hoek, E. M. A review of water treatment membrane nanotechnologies. Energy \& Environmental Science 4, 1946-1971, https://doi.org/10.1039/C0EE00541J (2011).

10 Sabir, A. et al. Conjugation of silica nanoparticles with cellulose acetate/polyethylene glycol 300 membrane for reverse osmosis using MgSO4 solution. Carbohydrate polymers 136, 551-559, https://doi.org/10.1016/j.carbpol.2015.09.042 (2016).

11 Fathizadeh, M., Aroujalian, A. \& Raisi, A. Effect of added NaX nano-zeolite into polyamide as a top thin layer of membrane on water flux and salt rejection in a reverse osmosis process. Journal of membrane science 375, 88-95, https://doi.org/10.1016/j.memsci.2011.03.017 (2011).

12 Ali, A. S. M., Soliman, M. M., Kandil, S. H. \& Khalil, M. M. Emerging mixed matrix membranes based on zeolite nanoparticles and cellulose acetate for water desalination. Cellulose, 1-10, https://doi.org/10.1007/s10570-021-03924-5 (2021).

13 Celik, E., Park, H., Choi, H. \& Choi, H. Carbon nanotube blended polyethersulfone membranes for fouling control in water treatment. Water research 45, 274-282, https://doi.org/10.1016/j.watres.2010.07.060 (2011).

14 Xia, S. \& Ni, M. Preparation of poly (vinylidene fluoride) membranes with graphene oxide addition for natural organic matter removal. Journal of Membrane Science 473, 54-62, https://doi.org/10.1016/j.memsci.2014.09.018 (2015).

15 Arsuaga, J. M. et al. Influence of the type, size, and distribution of metal oxide particles on the properties of nanocomposite ultrafiltration membranes. Journal of membrane science 428, 131-141, https://doi.org/10.1016/j.memsci.2012.11.008 (2013).

16 Mierzwa, J. C., Arieta, V., Verlage, M., Carvalho, J. \& Vecitis, C. D. Effect of clay nanoparticles on the structure and performance of polyethersulfone ultrafiltration membranes. Desalination 314, 147-158, https://doi.org/10.1016/j.desal.2013.01.011 (2013). 
17 Shi, F., Ma, Y., Ma, J., Wang, P. \& Sun, W. Preparation and characterization of $\mathrm{PVDF} / \mathrm{TiO}_{2}$ hybrid membranes with different dosage of nano- $\mathrm{TiO}_{2}$. Journal of Membrane Science 389, 522-531, https://doi.org/10.1016/j.memsci.2011.11.022 (2012).

18 Arthanareeswaran, G., Devi, T. S. \& Raajenthiren, M. Effect of silica particles on cellulose acetate blend ultrafiltration membranes: Part I. Separation and Purification Technology 64, 38-47, https://doi.org/10.1016/j.seppur.2008.08.010 (2008).

19 Kango, S. et al. Surface modification of inorganic nanoparticles for development of organic-inorganic nanocomposites-A review. Progress in Polymer Science 38, 12321261, https://doi.org/10.1016/j.progpolymsci.2013.02.003 (2013).

20 Wen, Y., Yuan, J., Ma, X., Wang, S. \& Liu, Y. Polymeric nanocomposite membranes for water treatment: a review. Environmental Chemistry Letters 17, 1539-1551, https://doi.org/10.1007/s10311-019-00895-9 (2019).

21 Ahmad, A. et al. Effect of silica on the properties of cellulose acetate/polyethylene glycol membranes for reverse osmosis. Desalination 355, 1-10, https://doi.org/10.1016/j.desal.2014.10.004 (2015).

22 Rahman, I. A. \& Padavettan, V. Synthesis of silica nanoparticles by sol-gel: size-dependent properties, surface modification, and applications in silica-polymer nanocomposites-a review. Journal of Nanomaterials 8, https://doi.org/10.1155/2012/132424 (2012).

23 Ali, A. S. M., Fadl, E. A., Soliman, M. M. \& Kandil, S. H. Optimization of the evaporation step in cellulose acetate membranes preparation by dry-wet phase inversion technique for water desalination applications. DESALINATION AND WATER TREATMENT 174, 6370, doi: 10.5004/dwt.2020.24862 (2020).

24 Rida, M. A. \& Harb, F. Synthesis and characterization of amorphous silica nanoparitcles from aqueous silicates uisng cationic surfactants. Journal of Metals, Materials and Minerals 24 (2014).

$25 \mathrm{Wu}, \mathrm{H}$., Tang, B. \& Wu, P. Novel hollow mesoporous silica spheres/polymer hybrid membrane for ultrafiltration. The Journal of Physical Chemistry C 116, 2246-2252, https://doi.org/10.1021/jp2073045 (2012).

26 Rafiee, E., Shahebrahimi, S., Feyzi, M. \& Shaterzadeh, M. Optimization of synthesis and characterization of nanosilica produced from rice husk (a common waste material). International nano letters 2, 29, https://doi.org/10.1186/2228-5326-2-29 (2012).

27 Beganskienè, A., Sirutkaitis, V., Kurtinaitienè, M., Juškėnas, R. \& Kareiva, A. FTIR, TEM and NMR Iinvestigations of Stöber Silica Nanoparticles. (2004).

28 Pijarn, N., Jaroenworaluck, A., Sunsaneeyametha, W. \& Stevens, R. Synthesis and characterization of nanosized-silica gels formed under controlled conditions. Powder Technology 203, 462-468, https://doi.org/10.1016/j.powtec.2010.06.007 (2010).

29 Vansant, E. F., Van Der Voort, P. \& Vrancken, K. C. Characterization and chemical modification of the silica surface. Vol. 93 (Elsevier, 1995).

30 Ma, X.-k. et al. Surface modification and characterization of highly dispersed silica nanoparticles by a cationic surfactant. Colloids and Surfaces A: Physicochemical and Engineering Aspects 358, 172-176, https://doi.org/10.1016/j.colsurfa.2010.01.051 (2010).

31 Xie, K., Yu, Y. \& Shi, Y. Synthesis and characterization of cellulose/silica hybrid materials with chemical crosslinking. Carbohydrate Polymers 78, 799-805, https://doi.org/10.1016/j.carbpol.2009.06.019 (2009).

$32 \mathrm{Gu}, \mathrm{H}$. et al. Study of amino-functionalized mesoporous silica nanoparticles (NH2-MSN) and polyamide-6 nanocomposites co-incorporated with NH2-MSN and organo- 
montmorillonite. Microporous and Mesoporous Materials 170, 226-234, https://doi.org/10.1016/j.micromeso.2012.12.010 (2013).

33 Sabir, A. et al. Novel polymer matrix composite membrane doped with fumed silica particles for reverse osmosis desalination. Desalination 368, 159-170, https://doi.org/10.1016/j.desal.2014.12.041 (2015).

34 Gupta, V. K., Pathania, D., Singh, P., Rathore, B. S. \& Chauhan, P. Cellulose acetatezirconium (IV) phosphate nano-composite with enhanced photo-catalytic activity. Carbohydrate polymers 95, 434-440, https://doi.org/10.1016/j.carbpol.2013.02.045 (2013).

35 Ali, M., Zafar, M., Jamil, T. \& Butt, M. T. Z. Influence of glycol additives on the structure and performance of cellulose acetate/zinc oxide blend membranes. Desalination 270, 98104, https://doi.org/10.1016/j.desal.2010.11.027 (2011).

36 Dehkordi, F. S., Pakizeh, M. \& Namvar-Mahboub, M. Properties and ultrafiltration efficiency of cellulose acetate/organically modified $\mathrm{Mt}$ (CA/OMMt) nanocomposite membrane for humic acid removal. Applied Clay Science 105, 178-185, https://doi.org/10.1016/j.clay.2014.11.042 (2015).

37 Belal, A. S., Khalil, M., Soliman, M. \& Ebrahim, S. Novel superhydrophobic surface of cotton fabrics for removing oil or organic solvents from contaminated water. Cellulose 27, 7703-7719, https://doi.org/10.1007/s10570-020-03316-1 (2020).

38 Taha, A. A., Wu, Y.-n., Wang, H. \& Li, F. Preparation and application of functionalized cellulose acetate/silica composite nanofibrous membrane via electrospinning for $\mathrm{Cr}$ (VI) ion removal from aqueous solution. Journal of environmental management 112, 10-16, https://doi.org/10.1016/j.jenvman.2012.05.031 (2012).

39 Chen, W. et al. In situ generated silica nanoparticles as pore-forming agent for enhanced permeability of cellulose acetate membranes. Journal of Membrane Science 348, 75-83, https://doi.org/10.1016/j.memsci.2009.10.042 (2010).

40 Sile-Yuksel, M., Tas, B., Koseoglu-Imer, D. Y. \& Koyuncu, I. Effect of silver nanoparticle (AgNP) location in nanocomposite membrane matrix fabricated with different polymer type on antibacterial mechanism. Desalination 347, 120-130, https://doi.org/10.1016/j.desal.2014.05.022 (2014).

41 Alpatova, A. et al. Fabrication of porous polymeric nanocomposite membranes with enhanced anti-fouling properties: Effect of casting composition. Journal of membrane science 444, 449-460, https://doi.org/10.1016/j.memsci.2013.05.034 (2013).

42 Arthanareeswaran, G., Devi, T. S. \& Mohan, D. Development, characterization and separation performance of organic-inorganic membranes: part II. Effect of additives. $\begin{array}{llll}\text { Separation } & \text { and } & \text { Purification 271-281, }\end{array}$ https://doi.org/10.1016/j.seppur.2009.03.037 (2009).

$43 \mathrm{He}, \mathrm{W}$. et al. Surface modification of colloidal silica nanoparticles: Controlling the size and grafting process. Bulletin of the Korean Chemical Society 34, 2747-2752, http://dx.doi.org/10.5012/bkcs.2013.34.9.2747 (2013).

44 Pendergast, M. T. M., Nygaard, J. M., Ghosh, A. K. \& Hoek, E. M. Using nanocomposite materials technology to understand and control reverse osmosis membrane compaction. Desalination 261, 255-263, https://doi.org/10.1016/j.desal.2010.06.008 (2010). 